\title{
A Method to Collect an Infrared Spectrum in Solution
}

\author{
Hai-shui WANG, ${ }^{\dagger}$ Fei Lu, and Hongju ZhaI \\ Changchun Institute of Applied Chemistry, Chinese Academy of Sciences, 5625 Renmin Street, \\ Changchun 130022, China
}

\begin{abstract}
Herein we report a new method to collect a qualified infrared spectrum of a solute in solution by two solvent cells with different thickness during background single-beam spectrum scanning. By collecting the background spectrum with two cells (two stages), we successfully achieved accurate solvent compensation between a sample and a reference, namely, the solvent amounts in the sample and background measurements could become congruent. Therefore, the solvent bands were thoroughly suppressed in the infrared spectrum and a qualified spectrum of the solute was obtained.
\end{abstract}

(Received February 27, 2007; Accepted May 31, 2007; Published July 10, 2007)

Most samples (gaseous, liquid or solid) can be prepared as solutions in appropriate solvents, and the infrared (IR) spectra in solutions have been widely obtained and studied. ${ }^{1-3}$ Solvents usually have absorptions in the infrared region, and their bands may overlap with the areas that contain structural information of the solute molecules. Therefore, the influence of the solvent has to be compensated for in a suitable manner. One solution to this problem is a suitable choice of the solvent; for example, the solvent should have a very simple IR spectrum with bands in the fingerprint region of the spectrum. Carbon tetrachloride and carbon disulfide are common IR solvents, which are often used as a solvent pair. They are used by scanning a solution of the sample in carbon tetrachloride from 4000 to $1350 \mathrm{~cm}^{-1}$ and a second solution in carbon disulfide from 1350 to $500 \mathrm{~cm}^{-1}$. This combination allows the observations of all the bands in an unknown spectrum without any significant interference from the solvent. Another widely used data manipulation technique in contemporary infrared spectrometry is spectral subtraction. This technique subtracts the absorbance spectrum of the solvent suitably scaled to obtain the best compensation of the solvent bands from the absorbance spectrum of solution. ${ }^{4,5}$

As we know, the best final infrared spectrum should be devoid of all instrumental and environmental contributions, and only present the features of the sample. If the amounts of solvent in the instrument are the same when the background and sample single beam spectra are obtained, the solvent contributions to the spectrum will ration out exactly and their bands will not occur. If the amounts of solvent are different when the background and sample spectra are obtained, solvent bands will appear in the final sample spectrum. Commonly used solvents in IR solution cells are $\mathrm{CCl}_{4}, \mathrm{CS}_{2}$, and $\mathrm{CHCl}_{3}$; they are usually transparent in the important absorption region of the spectrum. Therefore, a few path-length difference of the solvent between the background and the sample is allowed, and significant interference from the solvent can be avoided.

However, many organic compounds and polymers have poor solubility in IR solvents, such as $\mathrm{CCl}_{4}, \mathrm{CS}_{2}$. The frequently quoted rule "like dissolves like" can serve as a useful guide to choose a solvable solvent; for instance, benzene is readily soluble in toluene. If the solute and the solvent belong to the

$\doteqdot$ To whom correspondence should be addressed.

E-mail: hswhsw2000@yahoo.com.cn "like dissolves like" group, the solvent interference may be very severe in the infrared spectrum. In such a case, solvent bands should be removed from the spectrum. However, until now the perfect solvent compensation has not been achieved between the sample and the background (reference) because the dissolved material (solute) occupies some volume in the IR solution cell and the necessary accurate control of the cell thickness is limited. The purpose of this article is to provide a new way to accurately control the amounts of solvent in sample and reference measurements, and to collect a qualified infrared spectrum without any solvent interference.

\section{Experimental}

\section{Reagents and chemicals}

Benzene and toluene were obtained from Aldrich and used without further purification. A solution was prepared by mixing benzene and toluene in a suitable volume ratio.

\section{Apparatus}

A demountable liquid cell was purchased from Pike Technologies. It consisted of two $\mathrm{NaCl}$ disks, $32 \mathrm{~mm}$ in diameter, separated by a Teflon spacer. A $25-\mu \mathrm{m}$ thick spacer was used to contain the solution in the cell for sample measurements, and two spacers with thicknesses of 15 and $25 \mu \mathrm{m}$ for background measurements. Infrared spectra were measured at a $4-\mathrm{cm}^{-1}$ resolution with a Nicolet Magna 760 FT-IR spectrometer equipped with a DTGS detector. Each IR spectrum during each scan of multi-scanning procedure could be displayed; namely, the monitor could display each scanning spectrum in real time. Omnic software (Nicolet) usually provides a pause function during the scanning procedure. We use this pause function to replace one background cell by another one.

\section{Results and Discussion}

Solvent peaks can appear or disappear in the infrared spectrum depending on the experimental conditions. Upward peaks appear if the sample single beam spectrum contains more solvent molecules than the background spectrum, while downward peaks appear if the sample spectrum contains fewer 


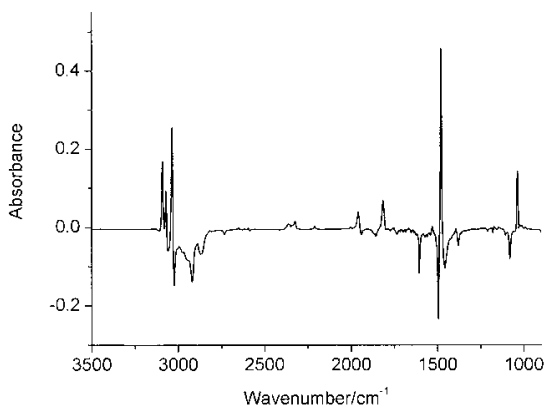

Fig. 1 Infrared spectrum of a toluene/benzene solution. Downward peaks are toluene bands.

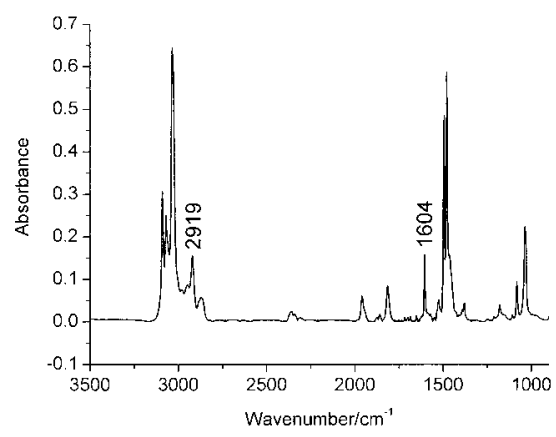

Fig. 2 Infrared spectrum of a toluene/benzene solution. Toluene bands are upward.

solvent molecules than the background spectrum. Figures 1 and 2 show infrared spectra of benzene/toluene solutions. Toluene bands appear as downward (Fig. 1) or upward (Fig. 2) features because the background (solvent) cell contains more or less toluene molecules than the solution cell. If solvent molecules become congruent between the sample and background measurements, solvent peaks should disappear accordingly.

We suppose that the path-length of the solvent in the solution cell is $b_{\mathrm{S}}$ and that the path-length in background cell is $b_{\mathrm{B}}$. If only one background cell and one sample cell are used, then that $b_{\mathrm{S}}$ equals $b_{\mathrm{B}}$ may be very difficult and only appears very occasionally, because the solute occupies volume in the IR solution cell. Here, we suggested a new idea to solve this problem. In the infrared spectrum measurement, we may use two background cells with thicknesses of $b_{\mathrm{B} 1}$ and $b_{\mathrm{B} 2}$, and make $b_{\mathrm{B} 1}>b_{\mathrm{S}}>b_{\mathrm{B} 2}$. The scanning number for cell one $\left(b_{\mathrm{B} 1}\right)$ is $N_{1}$ and that for cell two $\left(b_{\mathrm{B} 2}\right)$ is $N_{2}$; then, the mean path length is $\left(N_{1} b_{\mathrm{B} 1}+N_{2} b_{\mathrm{B} 2}\right) /\left(N_{1}+N_{2}\right)$. If $b_{\mathrm{S}}=\left(N_{1} b_{\mathrm{B} 1}+N_{2} b_{\mathrm{B} 2}\right) /\left(N_{1}+N_{2}\right)$, the solvent bands will be suppressed in the final spectrum. We have adopted the following steps to realize the above purpose. First, the sample single-beam spectrum (benzene/toluene solution) is measured with a cell thickness of $25 \mu \mathrm{m}$. In this experiment, we used two cells with thickness 15 and $25 \mu \mathrm{m}$ for background measurements. Therefore, the percentage concentration of toluene $(\mathrm{v} / \mathrm{v})$ should be controlled so that the path-length for the solvent (toluene) in the solution cell is greater than $15 \mu \mathrm{m}$. Next, the temporary background spectrum is measured with the toluene cell (thickness $15 \mu \mathrm{m}$ ) by scanning number 48 , and then pause the scanning procedure. Upward features of toluene will appear by observing the real-time display spectrum. The strong and independent IR band of the solvent should be selected as an internal intensity standard. In

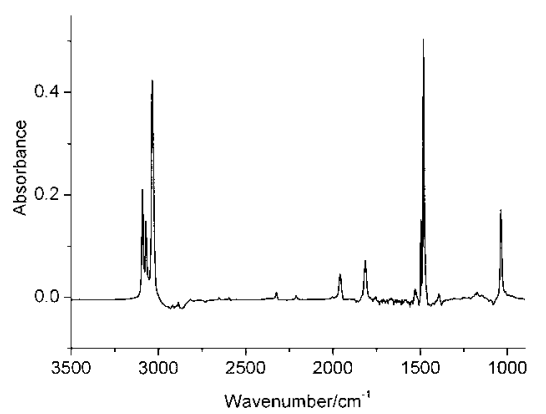

Fig. 3 Infrared spectrum of a toluene/benzene solution collected by the two background cells method. Toluene bands are thoroughly suppressed.

the present case, the $1604 \mathrm{~cm}^{-1}$ vibration (quadrant stretching) of toluene shown in Fig. 2 was selected. Then, place the toluene cell with a thickness of $25 \mu \mathrm{m}$ into the light-path, and continue the background scanning. The mean light-path in the background is $b_{\mathrm{mb}}=\left(48 \times 15+N_{2} \times 25\right) /\left(48+N_{2}\right)$, and when $N_{2}$ (scan number for $25 \mu \mathrm{m}$ cell in background) reaches a certain value, $b_{\mathrm{mb}}$ will approximate exactly to $b_{\mathrm{S}}$. The amount of the toluene in infrared spectrum can be easily viewed by the realtime display system during each background scan. After the 15 $\mu \mathrm{m}$ cell is replaced by a $25 \mu \mathrm{m}$ cell, the upward peaks of toluene in the infrared spectrum will become smaller and smaller with an increase in the scanning number, until they become unobservable $\left(b_{\mathrm{mb}} \rightarrow b_{\mathrm{s}}\right)$. Figure 3 shows the infrared spectrum that was obtained by scanning the $15 \mu \mathrm{m}$ cell 48 times and scanning the $25 \mu \mathrm{m}$ cell 36 times. When the total scanning number for background reaches 84 , the toluene bands disappear. We can stop the collection of the spectrum at any scanning number when the toluene bands are suppressed. The resultant spectrum in Fig. 3 indicates that the vibrations from toluene, such as the 1604 and $2919 \mathrm{~cm}^{-1}\left(-\mathrm{CH}_{3}\right.$ stretching) bands, are removed. Thus, the conclusion that an infrared spectrum free from solvent noise can be obtained by our method could be reached. Moreover, if there exists one independent and strong/middle band of the solvent in the spectrum, this method also works well where the bands of the solvent are highly overlapped with those of the solute.

\section{Acknowledgements}

This work was supported by National Natural Sciences Foundation of China (Nos. 20273068 and 90306001).

\section{References}

1. J. M. Chalmers and P. R. Griffiths, "Handbook of Vibrational Spectroscopy", 2002, John Wiley and Sons, New York.

2. H. A. Willis, J. H. Van Der Maas, and G. G. J. Miller, "Laboratory Methods in Vibrational Spectroscopy", 1987, John Wiley and Sons, New York.

3. N. B. Colthup, L. H. Daly, and S. E. Wiberley, "Introduction to Infrared and Raman Spectroscopy", 1990, Academic Press, San Diego.

4. K. Rahmelow and W. Hubner, Appl. Spectrosc., 1997, 51(2), 160.

5. K. Murayama and M. Tomida, Biochemistry, 2004, 43, 11526. 